УДК 621.315 .592

\title{
Нетривиальная зависимость спектральных характеристик экситонов в квантовых ямах от мощности резонансного оптического возбуждения
}

\author{
() Д.Ф. Мурсалимов ${ }^{1}$, А.В. Михайлов ${ }^{1}$, А.С. Курдюбов ${ }^{1}$, А.В. Трифонов ${ }^{1,2}$, И.В. Игнатьев ${ }^{1}$ \\ ${ }^{1}$ Санкт-Петербургский государственный университет, \\ 198504 Санкт-Петербург, Россия \\ 2 Технический университет Дортмунда, \\ 44221 Дортмунд, Германия \\ E-mail: d.mursalimov@yahoo.com
}

Поступила в Редакцию 12 апреля 2021 г.

В окончательной редакции 19 апреля 2021 г.

Принята к публикации 19 апреля 2021 г.

В гетероструктуре с квантовой ямой GaAs/AlGaAs шириной 14 нм экспериментально исследованы основные спектральные характеристики экситонных линий - энергия экситонного резонанса, а также его радиационное и нерадиационное уширения. Особое внимание уделено нерадиационному уширению как наиболее чувствительному к концентрации свободных носителей и долгоживущих экситонов. Наблюдается сублинейный рост уширения резонансов тяжелого и легкого экситонов с ростом мощности возбуждения в легкий экситон. Развита простая модель, позволяющая воспроизвести наблюдаемую зависимость.

Ключевые слова: экситон, квантовая яма, нерадиационное уширение.

DOI: $10.21883 /$ FTP.2021.11.51547.43

\section{1. Введение}

В настоящее время технологии выращивания гетероструктур на основе GaAs позволяют получать образцы исключительно высокого качества. В таких структуpax возможно исследование фундаментальных характеристик экситонов, таких как энергия экситонных переходов, сила свето-экситонного и экситон-фононного взаимодействий и т. д. Такие исследования важны как для проверки моделей экситонов в гетероструктурах с квантовыми ямами (КЯ), так и для уточнения параметров этих моделей. Дальнейшим развитием может служить использование экситона как чувствительного зонда для гетероструктур с КЯ [1].

Оптическое возбуждение помимо свободных, светоизлучающих экситонов способно рождать и другие квазичастицы, такие как свободные электроны и дырки, неизлучающие экситоны, трионы, биэкситоны и другие связанные состояния частиц. Как следствие, экситоны, наблюдаемые в спектрах отражения или фотолюминесценции (ФЛ), не являются свободными (изолированными), поскольку они испытывают влияние со стороны других квазичастиц, созданных оптическим возбуждением. Ограничить их влияние можно путем уменьшения мощности оптического возбуждения. Тем не менее даже при малой мощности нерезонансного возбуждения, необходимой для наблюдения экситонной ФЛ, образуется резервуар свободных носителей и долгоживущих экситонов [2]. Последние распространяются вдоль слоя КЯ, с волновым вектором $K$, превышающим волновой вектор света $K_{\text {c }}$ в материале КЯ. Такие экситоны не взаимодействуют со светом (темные и неизлучающие экситоны) [3-5], и в высококачественных гетероструктурах времена их жизни могут составлять десятки наносекунд [2]. В то же время для светлых экситонов, с $K<K_{\text {c, }}$ характерное время рекомбинации измеряется десятками пикосекунд [6]. Значительное различие времен жизни приводит к тому, что двумерная плотность неизлучающих экситонов в резервуаре на несколько порядков превышает плотность излучающих экситонов. Взаимодействие фоторожденных экситонов с резервуаром в значительной степени влияет на их спектральные характеристики. В частности, экситон-экситонное рассеяние приводит либо к прерыванию процесса рекомбинации электронно-дырочной пары, либо к изменению фазы излучающего состояния [7], что проявляется в уширении экситонных резонансов.

В нашей работе экспериментально исследовались свойства экситонов в GaAs-гетероструктуре методом спектроскопии отражения. Измерены мощностные зависимости основных спектральных характеристик экситонов при резонансном оптическом возбуждении. Развита простая модель для описания наблюдаемой экспериментально сублинейной зависимости уширения экситонного резонанса от мощности возбуждения.

\section{2. Эксперимент и обработка результатов}

Исследуемая структура была выращена методом молекулярно-пучковой эпитаксии (МПЭ) на GaAs-подложке $n$-типа проводимости с кристаллографической ориентацией (001). Гетероструктура включает КЯ шириной 


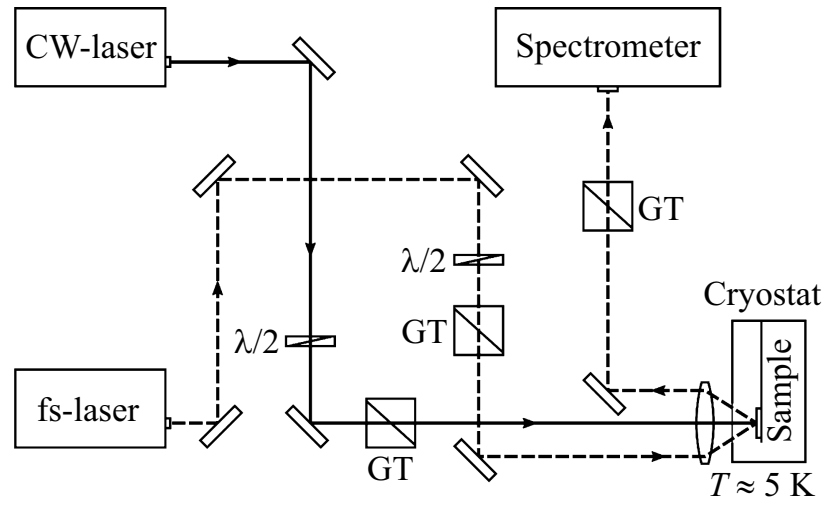

Рис. 1. Экспериментальная установка: fs-laser - импульсный лазер, CW-laser - фемтосекундный лазер, GT - призма Глана-Тейлора.

14 нм, выращенную между барьерами $\mathrm{Al}_{x} \mathrm{Ga}_{1-x} \mathrm{As}$ c небольшой концентрацией алюминия, $x \approx 3 \%$. Высокое качество образца обусловлено низким содержанием алюминия, минимизирующим встроенные деформации [1]. Образец охлаждался в криостате замкнутого цикла до температуры $T \approx 5 \mathrm{~K}$.

Спектры отражения измерялись при небольшом угле падения зондирующего луча к поверхности образца. Для зондирования использовались спектрально-широкие импульсы титан-сапфирового (Ti:sapphire) лазера длительностью 100 фс. Спектр отраженного излучения анализировался с помощью спектрометра с дифракционной решеткой 1800 штрихов/Мм и фокусным расстоянием 550 мм, детектировался матричным фотоприемником (ПЗС-матрица), охлаждаемым жидким азотом. Схема экспериментальной установки представлена на рис. 1. Для создания резервуара неизлучающих экситонов в наших экспериментах использовалось дополнительное оптическое возбуждение (накачка) излучением непрерывного титан-сапфирового лазера, резонансным с оптическим переходом в легкий экситон в исследуемой КЯ. Накачка проводилась при нормальном падении луча, поляризованного ортогонально лучу зондирования. Перед входом в спектрометр размещалась призма Глана-Тейлора, препятствующая попаданию рассеянного света от луча накачки на ПЗС-матрицу. Небольшой остаточный сигнал от рассеянного света луча накачки накапливали отдельно от зондирующего луча и вычитали при каждом измерении спектра. Проведенные таким образом измерения отличаются высокой надежностью и воспроизводимостью. Мощности лазерных пучков регулировались поворотом фазовых пластинок $\lambda / 2$, расположенных перед призмами Глана-Тейлора.

Спектр зондирующего луча, отраженного от образца, представляет собой широкий контур, хорошо аппроксимируемый гауссовой функцией с полушириной на полувысоте 10 мэВ. На фоне этого контура наблюдаются узкие резонансы, соответствующие легкому (Xlh) и тяжелому (Xhh) экситонам. Спектр отражения образца может быть получен путем нормировки спектра отраженного луча на гауссов профиль зондирующего импульса с учетом коэффициента отражения от поверхности. Типичный спектр отражения приведен на рис. 2.

Форма экситонных линий в виде одиночных пиков обусловлена оптимальным выбором толщины верхнего барьерного слоя КЯ, $L_{\text {top }} \approx 230$ нм. Длина оптического пути в слое $L_{\text {орt }}=L_{\text {top }} n(\mathrm{GaAs}) \approx 830$ нм $(n-$ показатель преломления) близка к длинам волн, соответствующим частотам экситонных резонансов. В результате возникает конструктивная интерференция света, отраженного от слоя КЯ и поверхности образца.

Спектральная ширина резонансов крайне чувствительна к условиям оптического возбуждения. При малой мощности зондирующего луча, 1 мкВт на площадь лазерного пятна $10^{-4} \mathrm{~cm}^{2}$, в отсутствие накачки ширина резонансной линии Хhh составляет $\Delta E=185$ мкэВ, что говорит о высоком качестве образца. Дополнительная подсветка непрерывным лазером приводит к существенному уширению резонансов (см. рис. 2).

Форма экситонных резонансов может быть смоделирована в рамках теории нелокального диэлектрического отклика, описанной в монографии Е.Л. Ивченко [8]. Эта модель применялась для анализа экспериментальных данных во многих работах (см., например, [1,2,9-12]). Амплитудный коэффициент отражения от слоя КЯ вблизи частоты экситонного резонанса $\omega_{\mathrm{X}}$ определяется выражением

$$
r_{\mathrm{QW}}(\omega)=\frac{i \Gamma_{\mathrm{R}}}{\omega_{\mathrm{X}}-\omega-i\left(\Gamma_{\mathrm{R}}+\Gamma_{\mathrm{NR}}\right)}
$$

где $\Gamma_{\mathrm{R}}$ и $\Gamma_{\mathrm{NR}}-$ скорости излучательной и безызлучательной релаксации экситонов соответственно. Интенсивность отраженного излучения зависит также от амплитудного коэффициента отражения поверхности об-

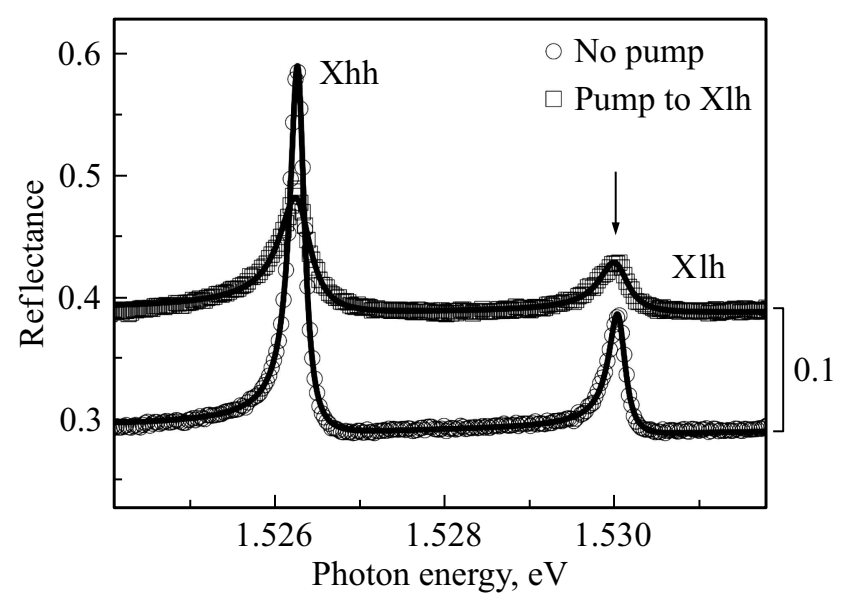

Рис. 2. Спектры отражения исследуемой структуры, измеренные без накачки (нижняя кривая) и при наличии дополнительного возбуждения в резонанс легкого экситона (верхняя кривая). Верхний спектр сдвинут на 0.1 для наглядного представления данных. Сплошные кривые - аппроксимации согласно формулам (1), (2). 
разца, $r_{\mathrm{s}}$, и выражается как

$$
R(\omega)=\left|\frac{r_{\mathrm{s}}+r_{\mathrm{QW}}(\omega) \mathrm{e}^{i 2 \phi}}{1+r_{\mathrm{s}} r_{\mathrm{QW}}(\omega) \mathrm{e}^{i 2 \phi}}\right|^{2},
$$

где $\phi-$ набег фазы световой волны при прохождении барьера до середины слоя КЯ.

Уравнения (1), (2) использовались для подгонки спектров, представленных на рис. 2. Видно, что аналитические кривые точно описывают все особенности резонансов. Следует отметить, что подгоночные кривые хорошо воспроизводят лоренцову форму резонансов с медленно затухающими крыльями, - это говорит об отсутствии заметного неоднородного уширения в системе. В противном случае поведение крыльев контура характеризовалось бы более быстрым спадом - гауссовой формой.

Хорошее согласие экспериментальных и расчетных кривых позволяет определить основные параметры экситонных резонансов: радиационное $\left(\hbar \Gamma_{\mathrm{R}}\right)$ и нерадиационное $\left(\hbar \Gamma_{\mathrm{NR}}\right)$ уширения, а также энергию экситона $\left(\hbar \omega_{\mathrm{X}}\right)$ с точностью от единиц до долей мкэВ. Следует отметить, что не исключены некоторые систематические ошибки в получаемых значениях подгоночных параметров, которые, однако, не зависят от условий оптического возбуждения и тем самым не препятствуют исследованию мощностных зависимостей. Для нижнего спектра (в отсутствие накачки) на рис. 2 параметры резонанса Xhh таковы: $\hbar \Gamma_{\mathrm{R}}=37$ мкэВ, $\hbar \Gamma_{\mathrm{NR}}=44$ мкэВ и $\hbar \omega_{\mathrm{X}}=1.526278$ эВ. Определение величины радиационного уширения $\hbar \Gamma_{\mathrm{R}}$ из эксперимента требует точного измерения коэффициента отражения, что вызывает трудности, поскольку поверхность образца не идеальна. Поэтому коэффициент отражения был выбран таким образом, чтобы величина радиационного уширения совпадала с результатами микроскопического расчета: $\hbar \Gamma_{\mathrm{R}}=37$ мкэВ $[12,13]$. Для резонанса Xlh энергия $\hbar \omega_{\mathrm{X}}=1.530056$ эВ и радиационное уширение $\hbar \Gamma_{\mathrm{R}}=15$ мкэВ, что в $\sim 2.5$ раза меньше, чем для Xhh.

Однородное нерадиационное уширение $\hbar \Gamma_{\mathrm{NR}}$ экситонных резонансов обусловлено взаимодействием (рассеянием) фоторожденных экситонов, созданных зондирующим лучом, с другими квазичастицами системы: свободными носителями, фононами, излучающими и неизлучающими экситонами $[1,2]$. Как видно из рис. 2 , такое взаимодействие приводит к существенному увеличению $\hbar \Gamma_{\mathrm{NR}}$ резонанса Xhh, достигающего 200 мкэВ при относительно небольшой плотности мощности накачки в $\mathrm{Xlh} 6 \mathrm{BT} \cdot \mathrm{cm}^{-2}$. Высокая чувствительность нерадиационного уширения к таким процессам рассеяния позволяет детально изучать экситон-экситонное взаимодействие.

Мы проанализировали параметры экситонных контуров при непрерывной накачке в резонанс Xlh. Для каждой мощности накачки были измерены спектры отражения и обработаны с помощью формул (1), (2). На рис. 3,4 представлены зависимости радиационного и нерадиационного уширений от мощности накачки для $\mathrm{Xhh}$ и Xlh соответственно.

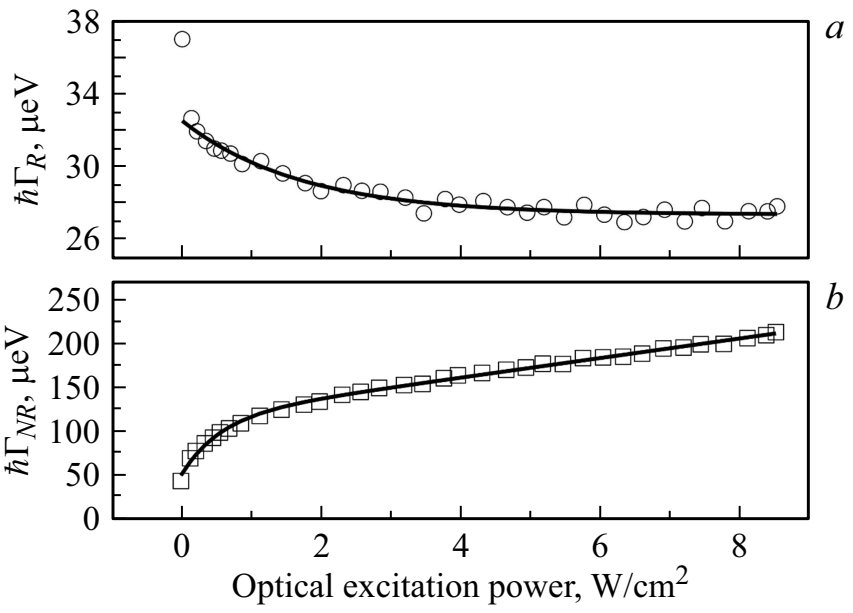

Рис. 3. Радиационное (a) и нерадиационное (b) уширения Xhh при непрерывном возбуждении в Xlh. Сплошная линия - аппроксимация феноменологической зависимостью $f=A\left[1-\exp \left(P_{\mathrm{exc}} / P_{0}\right)\right]+B P_{\mathrm{exc}}+C$.

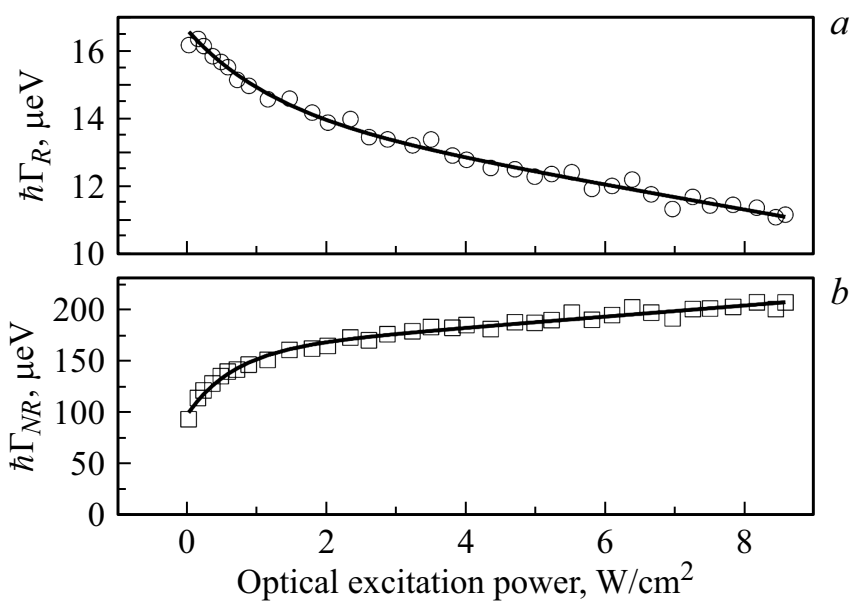

Рис. 4. Радиационное (a) и нерадиационное (b) уширения Xlh при непрерывном возбуждении в Xlh. Сплошная линия - аппроксимация феноменологической зависимостью $f=A 1-\exp \left[P_{\text {exc }} / P_{0}\right]+B P_{\text {exc }}+C$.

Как видно, параметры плавно меняются с ростом мощности накачки: радиационное уширение сублинейно падает, а нерадиационное сублинейно растет. Мы предполагаем, что такое поведение обусловлено взаимодействием светлых экситонов с экситонным резервуаром. Уменьшение радиационного уширения может быть связано с опустошением вакуумного состояния для экситонов за счет их накопления в резервуаре.

Плотность Xlh-экситонов, рожденных непрерывной накачкой, определяется плотностью мощности возбуждающих фотонов и коэффициентом поглощения $\alpha$ на частоте возбуждения. Последний может быть выражен как [8]

$$
\alpha_{\mathrm{Xlh}}(\omega)=\frac{2 \Gamma_{\mathrm{R}} \Gamma_{\mathrm{NR}}}{\left(\omega_{\mathrm{Xlh}}-\omega\right)^{2}+\left(\Gamma_{\mathrm{R}}+\Gamma_{\mathrm{NR}}\right)^{2}} .
$$




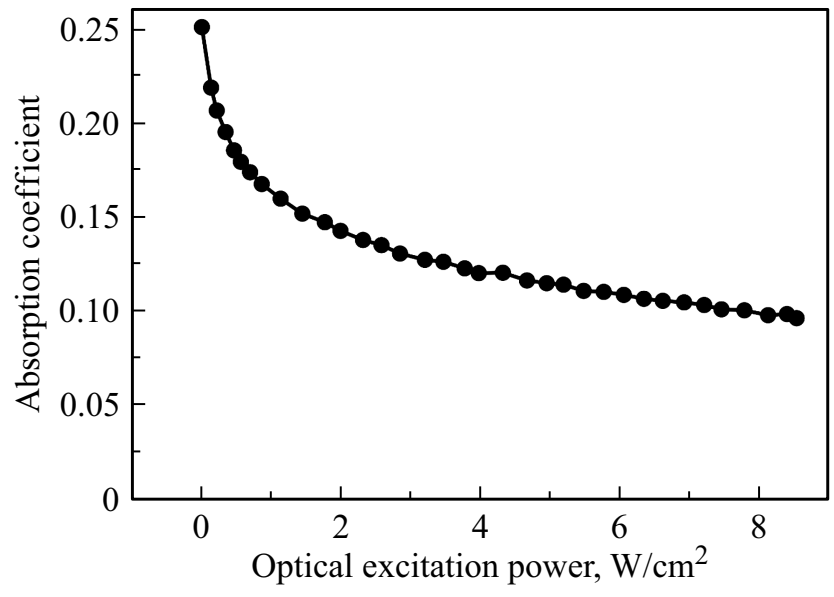

Рис. 5. Зависимость коэффициента поглощения в максимуме резонанса Xlh, вычисленная по формуле (3).

Подставляя мощностные зависимости уширений Xlh (рис. 4) в формулу (3), можно получить зависимость коэффициента поглощения в максимуме резонанса Xlh $\left(\alpha_{\mathrm{Xlh}}=\alpha_{\mathrm{Xlh}}\left(\omega_{\mathrm{Xlh}}\right)\right.$, см. рис. 5). Как видно, коэффициент поглощения значительно уменьшается с ростом мощности накачки в основном благодаря значительному увеличению $\hbar \Gamma_{\mathrm{NR}}$.

\section{3. Анализ мощностной зависимости нерадиационного уширения}

Мы провели анализ нерадиационного уширения в рамках модели динамических процессов, развитой в [14]. При резонансном возбуждении в резонанс легкого экситона рождаются излучающие экситоны, которые либо быстро релаксируют с испусканием фотона (скорость $\Gamma_{\mathrm{R}}$ ), либо рассеиваются за пределы светового конуса. В случае непрерывного возбуждения рассеяние обусловлено преимущественно испусканием акустических фононов (скорость $\gamma_{\text {phon }}$ ). Соотношение скоростей $f=\gamma_{\text {phon }} /\left(\Gamma_{\mathrm{R}}+\gamma_{\text {phon }}\right)$ определяет долю неизлучающих экситонов. Таким образом, скорость наполнения резервуара может быть записана как

$$
P_{\mathrm{XIh}}=\alpha_{\mathrm{Xlh}} N_{\text {phot }} f .
$$

Здесь $\quad N_{\text {phot }}=A P_{\text {exc }} /\left(\hbar \omega_{\text {Xih }}\right)-$ число фотонов $\left(\right.$ в $\left.\mathrm{cm}^{-2} \cdot \mathrm{c}^{-1}\right)$, достигших слоя КЯ, $P_{\text {ехс }}-$ мощность оптического возбуждения. Коэффициенты пропускания окна криостата и поверхности образца $-t_{w}$ и $t_{\mathrm{s}}$ соответственно - учтены в множителе $A=t_{w} t_{\mathrm{s}}$.

В результате рассеяния легкие экситоны могут либо перейти на дисперсионную ветвь тяжелых экситонов (скорость $\left.\gamma_{\mathrm{Xhh}}\right)$, либо диссоциировать на электроны и дырки (скорость $\gamma_{\mathrm{eh}}$ ). Последние в рассматриваемой модели играют важную роль: неизлучающие экситоны, рассеиваясь на свободных носителях, попадают обратно в световой конус, где рекомбинируют с испусканием фотонов. Этот бимолекулярный процесс приводит к опустошению экситонного резервуара и характеризуется скоростью $\kappa_{\mathrm{c}}$. В свою очередь свободные электроны и дырки могут объединяться в экситоны, тем самым восполняя резервуар (бимолекулярная скорость $\kappa_{\mathrm{ex}}$ ). В результате в системе устанавливается динамическое равновесие.

Динамика экситонов и носителей заряда в резервуаре описывается следующей системой уравнений:

$$
\left\{\begin{array}{l}
\frac{d n_{\mathrm{Xlh}}}{d t}=P_{\mathrm{Xlh}}-\left(\gamma_{\mathrm{Xhh}}+\gamma_{\mathrm{eh}}\right) n_{\mathrm{Xlh}} \\
\frac{d n_{\mathrm{e}}}{d t}=\frac{d n_{\mathrm{h}}}{d t}=\gamma_{\mathrm{eh}} n_{\mathrm{Xlh}}-\kappa_{\mathrm{eX}} n_{\mathrm{e}} n_{\mathrm{h}} \\
\frac{d n_{\mathrm{Xhh}}}{d t}=\gamma_{\mathrm{Xhh}} n_{\mathrm{Xlh}}+\kappa_{\mathrm{eX}} n_{\mathrm{e}} n_{\mathrm{h}}-\kappa_{\mathrm{c}} n_{\mathrm{Xhh}}\left(n_{\mathrm{e}}+n_{\mathrm{h}}\right)
\end{array} .\right.
$$

Здесь $n_{\mathrm{Xhh}}, n_{\mathrm{Xlh}}$ и $n_{\mathrm{e}}=n_{\mathrm{h}} \equiv n-$ двумерные плотности тяжелых, легких экситонов, а также свободных носителей в резервуаре соответственно.

В системе уравнений (5) не учтен возможный процесс повторного выбрасывания Хhh-экситонов из светового конуса в резервуар, поскольку излучательное время жизни этих экситонов чрезвычайно мало $(\sim 10$ пс). Соответственно их плотность на несколько десятичных порядков меньше плотности экситонов в резервуаре. В принципе такой процесс может идти за счет поглощения фононов или рассеяния экситонов свободными носителями. Оценки показывают, что оба механизма могут давать маленький дополнительный вклад в нерадиационное уширение (несколько процентов) при максимальной использованной мощности накачки.

В случае непрерывного возбуждения система уравнений (5) решается аналитически (производные по времени приравниваются нулю). Из первого уравнения получаем

$$
x_{\mathrm{Xlh}}=\frac{P_{\mathrm{Xlh}}}{\gamma_{\mathrm{Xhh}}+\gamma_{\mathrm{eh}}} .
$$

Из второго уравнения определим двумерную плотность свободных носителей:

$$
n=\sqrt{\frac{\gamma_{\mathrm{eh}} \kappa_{\mathrm{ex}}}{\kappa_{\mathrm{Xlh}}}}
$$

Тогда для двумерной плотности тяжелых экситонов в резервуаре из третьего уравнения имеем

$$
n_{\mathrm{Xhh}}=\frac{\gamma_{\mathrm{Xhh}}+\gamma_{\mathrm{eh}}}{2 \kappa_{\mathrm{c}} n} n_{\mathrm{Xlh}}=\frac{1}{2 \kappa_{\mathrm{c}}} \sqrt{\kappa_{\mathrm{ex}} \frac{\gamma_{\mathrm{Xhh}}+\gamma_{\mathrm{eh}}}{\gamma_{\mathrm{eh}}}} \sqrt{P_{\mathrm{Xlh}}} .
$$

Как видно из выражения (8), двумерная плотность тяжелых экситонов в резервуаре имеет корневую зависимость от скорости накачки $P_{\text {Xlh. Последняя в свою }}$ очередь нелинейно зависит от мощности оптического возбуждения $P_{\text {exc }}$ через коэффициент поглощения $\alpha_{\mathrm{Xlh}}-$ формула (4).

Нерадиационное уширение резонанса Xhh обусловлено взаимодействием фоторожденных экситонов со 


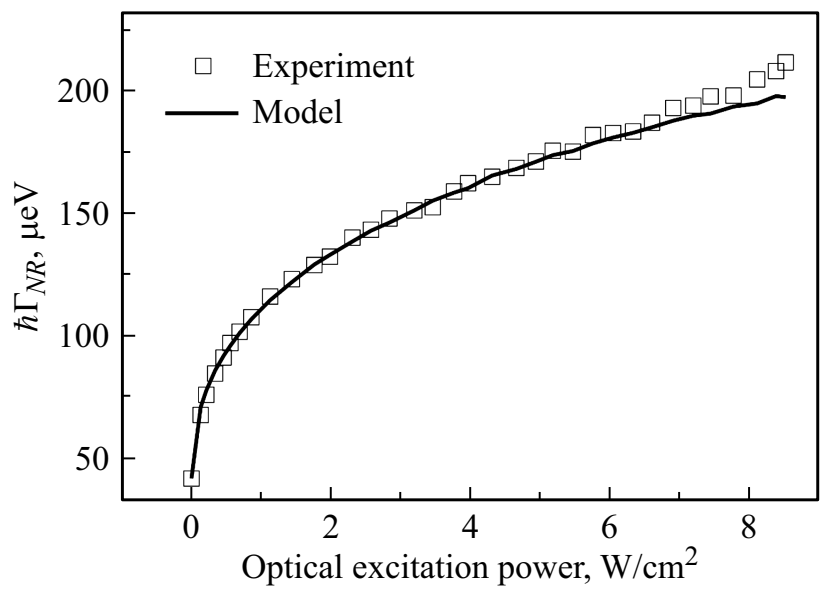

Рис. 6. Экспериментальная и теоретически рассчитанная зависимости нерадиационного уширения Xhh от мощности непрерывного возбуждения в Xlh.

всеми квазичастицами в резервуаре. Однако оценки показывают, что в текущих экспериментальных условиях двумерная плотность тяжелых экситонов в резервуаре значительно превышает (в десятки и сотни раз) плотности других частиц (легких экситонов, свободных носителей). Поэтому нерадиационное уширение преимущественно определяется двумерной плотностью тяжелых экситонов,

$$
\hbar \Gamma_{\mathrm{NR}} \approx n_{\mathrm{Xhh}} \sigma
$$

где $\sigma$ - сечение экситон-экситонного рассеяния. Воспользовавшись формулами (4), (8), (9) и предполагая, что $\sigma$ и константы скоростей не зависят от мощности возбуждения, получаем мощностную зависимость величины нерадиационного уширения (см. рис. 6). Видно, что теоретический расчет хорошо согласуется с экспериментом.

\section{4. Заключение}

В высококачественных гетероструктурах с КЯ резонансные линии тяжелого и легкого экситонов, наблюдаемые в спектрах отражения, могут быть достаточно точно описаны с привлечением теории нелокального диэлектрического отклика. В частности, отсутствие в системе источников неоднородного уширения позволяет определить основные параметры резонансов с точностью до долей мкэВ. Такая модельная система идеально подходит для исследований экситон-экситонного взаимодействия.

В рамках теории динамических процессов дана интерпретация сублинейного поведения нерадиационного уширения резонанса тяжелого экситона при увеличении мощности оптического возбуждения. Выполненный расчет показал полное согласие теоретической модели с экспериментом. Полученный результат указывает на то, что учтены все доминирующие процессы, и при данных экспериментальных условиях параметры модели не зависят от мощности возбуждения.

\section{Благодарности}

Авторы благодарят СПбГУ, грант № 73031758, и Российский фонд фундаментальных исследований, гранты № 19-02-00576а, № 20-32-70131, за финансовую поддержку. Авторы также благодарят Ресурсный центр „Нанофотоника“ СПбГУ за образец для исследований.

\section{Конфликт интересов}

Авторы заявляют, что у них нет конфликта интересов.

\section{Список литературы}

[1] P.Yu. Shapochkin, S.A. Eliseev, V.A. Lovtcius, Yu.P. Efimov, P.S. Grigoryev, E.S. Khramtsov, I.V. Ignatiev. Phys. Rev. Appl., 12, 034034 (2019).

[2] A.V. Trifonov, S.N. Korotan, A.S. Kurdyubov, I.Ya. Gerlovin, I.V. Ignatiev, Yu.P. Efimov, S.A. Eliseev, V.V. Petrov, Yu.K. Dolgikh, V.V. Ovsyankin, A.V. Kavokin. Phys. Rev. B, 91, 115307 (2015).

[3] M. Combescot, O. Betbeder-Matibet, R. Combescot. Phys. Rev. Lett., 99, 176403 (2007).

[4] M. Combescot, M.G. Moore, C. Piermarocchi. Phys. Rev. Lett., 106, 206404 (2011).

[5] J.O. Tollerud, S.T. Cundiff, J.A. Davis. Phys. Rev. Lett., 117, 097401 (2016).

[6] L.C. Andreani, F. Tassone, F. Bassani. Sol. St. Commun., 77 (9), 641 (1991).

[7] M.O. Scully, M.S. Zubairy. Quantum Optics (Cambridge, Cambridge University Press, 1997).

[8] E.L. Ivchenko. Optical Spectroscopy of Semiconductor Nanostructures (Berlin, Springer, 2004).

[9] A.V. Trifonov, E.S. Khramtsov, K.V. Kavokin, I.V. Ignatiev, A.V. Kavokin, Y.P. Efimov, S.A. Eliseev, P.Yu. Shapochkin, M. Bayer. Phys. Rev. Lett., 122, 147401 (2019).

[10] E.S. Khramtsov, P.A. Belov, P.S. Grigoryev, I.V. Ignatiev, S.Yu. Verbin, Yu.P. Efimov, S.A. Eliseev, V.A. Lovtcius, V.V. Petrov, S.L. Yakovlev. J. Appl. Phys., 119, 184301 (2016).

[11] P.S. Grigoryev, A.S. Kurdyubov, M.S. Kuznetsova, I.V. Ignatiev, Yu.P. Efimov, S.A. Eliseev, V.V. Petrov, V.A. Lovtcius, P.Yu. Shapochkin. Superlatt. Microstr., 97, 452 (2016).

[12] E.S. Khramtsov, P.S. Grigoryev, D.K. Loginov, I.V. Ignatiev, Yu.P. Efimov, S.A. Eliseev, P.Yu. Shapochkin, E.L. Ivchenko, M. Bayer. Phys. Rev. B, 99, 035431 (2019).

[13] P.A. Belov. J. Phys.: Conf. Ser., 1482, 012018 (2020).

[14] A.S. Kurdyubov, A.V. Trifonov, I.Ya. Gerlovin, B.F. Gribakin, P.S. Grigoryev, A.V. Mikhailov, I.V. Ignatiev, Yu.P. Efimov, S.A. Eliseev, V.A. Lovtcius, M. Assmann, M. Bayer, A.V. Kavokin. Phys. Rev. B 104 (3), 035414 (2021).

Редактор Л.В. Шаронова 


\title{
Non-trivial dependence of spectral characteristics of excitons in quantum wells on the resonant optical excitation power
}

D.F. Mursalimov' ${ }^{1}$, A.V. Mikhailov' ${ }^{1}$, A.S. Kurdyubov' ${ }^{1}$, A.V. Trifonov ${ }^{1,2}$, I.V. Ignatiev ${ }^{1}$

${ }^{1}$ Spin Optics Laboratory,

St. Petersburg State University, 198504 Peterhof, St. Petersburg, Russia

${ }^{2}$ Experimentelle Physik 2,

Technische Universitat Dortmund, 44221 Dortmund, Germany

\begin{abstract}
Basic exciton parameters, the energy of exciton transition and the radiative and nonradiative broadenings, are experimentally studied by means of reflectance spectroscopy for a heterostructure with the 14-nm GaAs/AlGaAs quantum well. Particular attention is paid to the nonradiative broadening which is sensitive to optical creation of free carriers and long-lived nonradiative excitons. A sublinear increase of the broadening of the heavy-hole and light-hole exciton resonances is observed when the light-hole exciton resonance is excited with increasing power. A simple model is developed, which allows one to well reproduce the observed dependence.
\end{abstract}

